\title{
Applicability of Rydberg atoms to quantum computers
}

\author{
Igor I. Ryabtsev $ه$ Denis B. Tretyakov, and Ilya I. Beterov \\ Institute of Semiconductor Physics \\ Prospekt Lavrentyeva 13, 630090 Novosibirsk, Russia
}

(Dated: January 30, 2004; revised May 14, 2004)

\begin{abstract}
Applicability of Rydberg atoms to quantum computers is examined from experimental point of view. In many theoretical proposals appeared recently, excitation of atoms into highly excited Rydberg states was considered as a way to achieve quantum entanglement in cold atomic ensembles via dipole-dipole interaction that could be strong for Rydberg atoms. Appropriate conditions to realize a conditional quantum phase gate have been analyzed. We also present the results of modeling experiments on microwave spectroscopy of single- and multi-atom excitations at the one-photon $37 \mathrm{~S}_{1 / 2} \rightarrow 37 \mathrm{P}_{1 / 2}$ and two-photon $37 \mathrm{~S}_{1 / 2} \rightarrow 38 \mathrm{~S}_{1 / 2}$ transitions in an ensemble of a few sodium Rydberg atoms. The microwave spectra were investigated for various final states of the ensemble initially prepared in its ground state. The quantum NOT operation with single atoms was found to be affected by the Doppler effect and fluctuations of the microwave field. The spectrum of full excitation of several Rydberg atoms was much narrower than that of a single atom. This effect might be useful for the high-resolution spectroscopy. The results may be also applied to the studies on collective laser excitation of ground-state atoms aiming to realize quantum gates.

PACS numbers: 03.67.Lx, 32.80.Rm, 32.70.Jz
\end{abstract}

\section{INTRODUCTION}

Quantum Computers $(\mathrm{QC})$ are of great interest last years [1]. Numerous proposals have been made on the practical realization of QC based on the NMR in complex molecules, cold ions in electrostatic traps, QED systems with high-Q cavities, and cold atoms trapped in optical lattices. The molecules, ions, and QED systems are well suited for modeling experiments demonstrating simple quantum operations on several qubits, but are difficult to scale to larger number of qubits required for a realistic QC [2]. More promising experiments could be made with the cold trapped neutral atoms having advantages of low decoherence rate, scalability to large number of qubits, and ability to control their states by individual addressing of resonant optical pulses.

Atoms in optical lattices are usually trapped in two or three dimensions at the antinodes of the standing light waves [3]. In other schemes implemented recently, atoms were trapped in the focuses of a two-dimensional array of microlenses spaced by $125 \mu \mathrm{m}$ [4], or in the focuses of two laser beams spaced by a few micrometers 5 . Ideally, each antinode should contain a single trapped atom representing a single qubit. Deterministic loading of single atoms into the focus is a delicate problem that was studied in Ref. [6].

The next stage is the operation on single or two qubits. Quantum computation can be performed using a sequence of single-qubit (NOT) and two-qubit (ControlledNOT, or CNOT) operations in an ensemble of qubits [7]. In the case of alkali atoms, a two-level qubit is represented by two hyperfine sublevels of a ground $\mathrm{S}$ state.

*Electronic address: ryabtsev@isp.nsc.ru
The NOT operator is implemented by Raman transitions between the sublevels, induced by a two-frequency laser pulse that inverts quantum state of qubit. The CNOT operator is much more difficult to realize, since it corresponds to a quantum nondemolition measurement of one ("control") qubit interacting with another ("target") qubit, and after its action the two qubits should be in an entangled state. The main experimental problem is thus to make entangled any of two atoms in the lattice, or at least any of two neighboring atoms.

Recently, the dipole-dipole interaction was proposed for use to entangle neutral alkali atoms in lattices or in cold atomic ensembles 8]. This interaction, however, is weak for the ground-state or low-excited atoms. In order to make the interaction stronger, it was proposed in Refs. [9, 10] to excite atoms for a while into the $n L$ Rydberg states with $n \gg 1$, and after some manipulations bring them back to ground state. Large atomic sizes (growing as $n^{2}$ ) and correspondingly large dipole moments of Rydberg atoms [1] strongly enhance the dipole-dipole interaction. This interaction changes the energies of Rydberg states, and may be viewed as a Stark shift in an atom placed in the dipole electric fields of other atoms. The energy of this atom thus depends on the states of neighboring atoms (whether they are in a Rydberg or in a ground state). Entangled states may be generated using a sequence of optical pulses applied to chosen qubits 9, 12, 13] or using dipole-induced changes in the spectra of collective excitations in the ensemble of Rydberg atoms ("dipole blockade") 10, 14.

Similar considerations were also made for the van der Waals (vdW) interaction between Rydberg atoms 15 . However, this interaction could be strong only for high Rydberg states and short interatomic distances comparable with the size of Rydberg atoms. In comparison with dipole-dipole interaction, it is advantageous due to inde- 
pendence of the orientation of dipoles, but seems to be impractical from its weakness. Nevertheless, experimental studies of the vdW interaction are of interest since it may affect the laser excitation of Rydberg states. The first experimental observation of vdW for Rydberg atoms in a dense atomic beam was reported in [16], and some experimental indications of the local vdW blockade in the ensemble of cold Rydberg atoms were reported recently [17.

Shifts and broadenings due to dipole-dipole interactions in large ensembles of cold Rydberg atoms in magneto-optical traps were observed for the Stark resonances in resonant collisions [18], and recently for the microwave transitions between Rydberg states [19]. However, experimental observation of dipole-induced frequency shifts of transitions for two Rydberg atoms or dipole blockade for a small ensemble have not been reported yet. Such experiments meet many technical difficulties, require a dense optical lattice with a detection system for Rydberg atoms and powerful narrowband lasers for fast excitation of Rydberg states from the ground state.

On the other hand, some modeling experiments with low number of atoms may be performed in an atomic beam with microwave transitions between neighboring Rydberg states. Such experiments can reveal important features of the multi-particle excitation spectra of Rydberg atoms.

In this paper we discuss the applicability of Rydberg atoms to QC from a practical point of view, and analyze conditions required to implement proposed schemes in practice. We also present the results of modeling experiments on the microwave spectroscopy of multi-atom excitations of sodium Rydberg atoms.

\section{EXPERIMENTAL LIMITATIONS ON QC WITH RYDBERG ATOMS}

\section{A. Principal quantum numbers: limitations by collisions}

The dipole moment of an atom is proportional to the average radius $r$ of the electron orbit. We have to choose $r$ as large as possible in order to use advantages of Rydberg atoms. In a hydrogen atom, $r$ is given by the formula [20]

$$
r=\frac{1}{2} a_{0}\left\{3 n^{2}-L(L+1)\right\}
$$

where $a_{0}$ is the Bohr radius. $r$ has close value also for the Rydberg states of hydrogen-like alkali atoms. A short distance $R$ between neighboring atoms in the lattice imposes obvious limitation on the maximal value of $r$ : $R$ must exceed $r$ by an order of magnitude to avoid collisions between electrons of close Rydberg atoms. Collisions have large cross-sections (comparable with atomic size), and may cause fast decoherence of Rydberg states (e.g., mixing of degenerate magnetic sublevels or stochastic jumps in the phases of wave-functions). Assuming $r<R / 10$, the maximum principal quantum number $n_{\max }$ of Rydberg atoms in the lattice is thus limited by

$$
n_{\max } \sim \sqrt{\frac{R}{15 a_{0}}+\frac{L(L+1)}{3}} .
$$

In the optical lattices of alkali atoms, $R$ can vary from $\sim 0.5 \mu \mathrm{m}$ (in red-detuned dipole traps) to $\sim 5 \mu \mathrm{m}$ (in quasi-electrostatic traps with a $\mathrm{CO}_{2}$-laser) [3], or to 125 $\mu \mathrm{m}$ for microlenses array [4]. The spacing $0.5 \mu \mathrm{m}$ is too small for reliable individual optical access to each qubit without disturbing the neighboring qubits, while $125 \mu \mathrm{m}$ is too long for effective dipole-dipole interaction (see the next subsection). The $5 \mu \mathrm{m}$ value seems to be optimal for both of optical access and compatibility with $\mathrm{CO}_{2}$-laser traps having an advantage of extremely low scattering rate of photons.

From Eq.(2) for S-states $(L=0)$ one obtains $n_{\max } \sim 70$ at $R \sim 5 \mu \mathrm{m}$. For circular Rydberg states [1] with maximum possible $L=(n-1)$, the estimate is $n_{\max } \sim 90$ at $R \sim 5$ $\mu \mathrm{m}$. Excitation of circular states, however, requires much time and sophisticated combination of the optical and microwave pulses in the magnetic and electric fields. One can conclude that in practice low- $L$ states with $n<70$ should be chosen, although higher $n$ would result in a stronger dipole interaction, and experiments with $n \sim 500$ have been already reported [21].

For further considerations we take $n<70$ and $R \simeq 5 \mu \mathrm{m}$ as preliminary parameters satisfying the following experimental conditions: (i) $R \simeq 5 \mu \mathrm{m}$ is a spacing that provides free optical access to each qubit and compatibility with $\mathrm{CO}_{2}$-laser lattices; (ii) $n \simeq 70$ is a maximal principal quantum number at given $R$ that ensures absence of collisions between neighboring Rydberg atoms.

\section{B. Dipole-dipole interaction and time of quantum operation}

Now we have to analyze the dipole-dipole interaction at the $n$ and $R$ values specified above. Consider the interaction between two identical Rydberg atoms $a$ and $b$ spaced by a distance $R$ (Fig.1). These two atoms may be viewed as a quasi-molecule whose energy eigenstates should be determined in a molecular wavefunctions basis $\left|\Psi_{a} \Psi_{b}\right\rangle$, as it was made in Refs. [8, 9, 10]. For example, if we choose for the consideration two close nondegenerate Rydberg states of opposite parity, $|1\rangle$ and $|2\rangle$, molecular states $|12\rangle$ and $|21\rangle$ are degenerate in energy and experience strong dipole-dipole interaction. The eigenstates of the quasi-molecule are readily found by solving the 


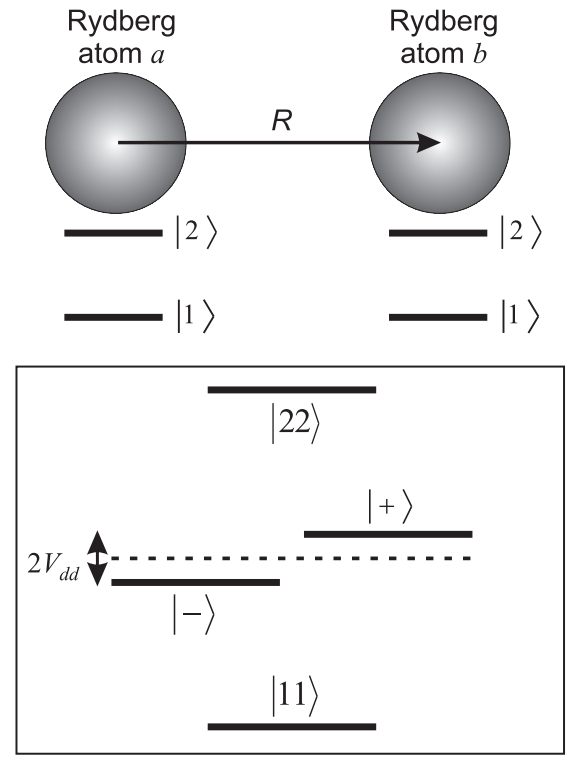

FIG. 1: A model for the dipole-dipole interaction between two Rydberg atoms. States $|1\rangle$ and $|2\rangle$ are two neighboring Rydberg levels. States $|11\rangle,|-\rangle,|+\rangle,|22\rangle$ are eigenstates of a composite system of two interacting atoms.

secular equation with the interaction Hamiltonian

$$
\hat{H}_{d d}=\frac{1}{4 \pi \varepsilon_{0}}\left[\frac{\hat{\mathbf{d}}_{a} \hat{\mathbf{d}}_{b}}{R^{3}}-\frac{3\left(\hat{\mathbf{d}}_{a} \mathbf{R}\right)\left(\hat{\mathbf{d}}_{b} \mathbf{R}\right)}{R^{5}}\right],
$$

where $\hat{\mathbf{d}}_{a}$ and $\hat{\mathbf{d}}_{b}$ are dipole moment operators of atoms $a$ and $b, \mathbf{R}$ is a vector connecting two atoms, and $\varepsilon_{0}$ is the dielectric constant. The solution shows that degenerate states of the quasi-molecule are split by the dipole-dipole interaction to two sublevels with symmetric (superradiant) and antisymmetric (subradiant) wave functions

$$
\begin{aligned}
& |+\rangle=\frac{1}{\sqrt{2}}(|12\rangle+|21\rangle), \\
& |-\rangle=\frac{1}{\sqrt{2}}(|12\rangle-|21\rangle) .
\end{aligned}
$$

If the quantization axis $z$ is chosen along $\mathbf{R}$, and states $|1\rangle$ and $|2\rangle$ have identical momentum projections that are bound only by the $\hat{d}_{z}$ components of dipole operators (e.g., particular Zeeman sublevels of states $|1\rangle$ and $|2\rangle$ whose degeneracy is lifted by a magnetic field), the energy shifts of these states are

$$
V_{d d}= \pm 2 d_{z}^{2} /\left(4 \pi \varepsilon_{0} R^{3}\right)
$$

where $d_{z}$ is $z$ component of the dipole matrix element of the $1 \rightarrow 2$ transition.

Addressing short individual laser pulses to the atoms, we can initially excite atom $a$ into state $|1\rangle$ and atom $b$ into state $|2\rangle$. Then atoms start to interact, and the time evolution of the wavefunction of the quasimolecule is following:

$$
\Psi(t)=|12\rangle \cos \left(V_{d d} t / \hbar\right)-i|21\rangle \sin \left(V_{d d} t / \hbar\right),
$$

i.e., population oscillates between states $|12\rangle$ and $|21\rangle$ with a frequency $\left(2 V_{d d} / \hbar\right)$.

This process may be considered as an exchange of virtual photons between two atoms. It was already observed experimentally when two Rb atoms in circular Rydberg states with $n=50$ and 51 were passed through a high-Q superconducting microwave cavity with variable detuning from resonance transition between these states 22]. The cavity strongly enhanced the interaction so that photon exchange occurred at $R \sim 1 \mathrm{~mm}$ on a time scale of $\sim 100$ $\mu \mathrm{s}$. Huge gain of the dipole-dipole interaction makes high-Q cavities attractive for modeling experiments on QC employing atoms passed through the cavity. However, in more realistic QC with cold trapped atoms it would complicate the loading of the lattice and optical access to atoms, and require special arrangement for cavity cooling to the temperatures $\sim 1 \mathrm{~K}$. Cooling is needed to achieve the superconductivity and, what is more important, to suppress the photons of blackbody radiation (BBR) that induce uncontrolled transitions between Rydberg states 11]. The cavity significantly increases rates of BBR-transitions, as we have demonstrated in [23], although usage of detuned cavity in 22] made atoms less sensitive to BBR. Further we will analyze the interaction in a free space, since practical application of a cavity for enhancement of dipole-dipole interaction is ambiguous.

From Eq. (6) one finds that in a free space the population returns back to state $|12\rangle$ after the time

$$
T=\pi \hbar / V_{d d},
$$

while the wavefunction changes its sign, or obtains a phase shift $\pi$. Such evolution is well suited for realization of a conditional Quantum Phase Gate (QPG) proposed in 24] and realized in a microwave cavity experiment with $\mathrm{Rb}$ Rydberg atoms [25]. QPG is of great importance since it may be consequently converted to CNOT gate with additional rotation of one of qubits. QPG was also analyzed theoretically in 12 for two Rb Rydberg atoms trapped in close laser focuses, as in experiment [5]. A scheme was considered in which two atoms were initially excited to the same $42 \mathrm{~S}$ Rydberg state, lying almost in the middle between two neighboring states $41 \mathrm{P}$ and $42 \mathrm{P}$. Similar situation was also employed in proposals on the dipole blockade at multi-atom excitations in the atomic ensemble, but in this case an electric field was required to tune transitions to exact resonance 10, 14.

We propose another scheme that uses only two $n \mathrm{~S}$ and $n \mathrm{P}$ Rydberg states in two alkali atoms $a$ and $b$ (Fig.2), and no Stark tuning is needed. The ground S-state has two hyperfine sublevels $\mathrm{F}=1$ and $\mathrm{F}=2$ (for example, in ${ }^{23} \mathrm{Na}$ and ${ }^{87} \mathrm{Rb}$ atoms) which represent two qubit states 

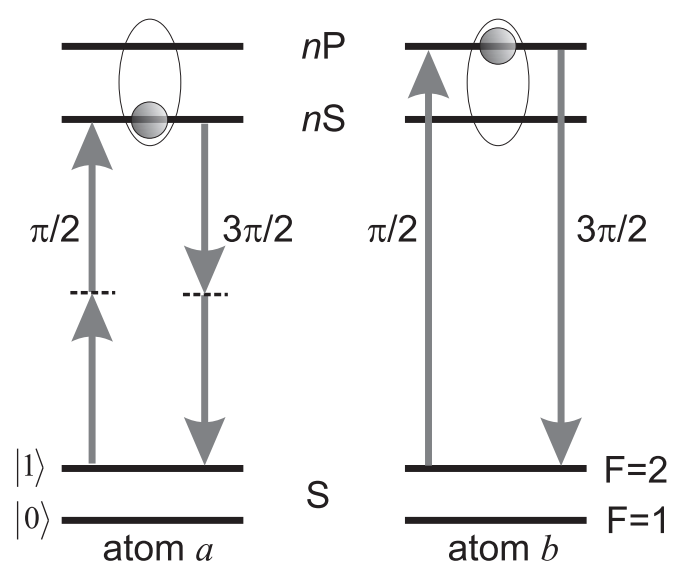

FIG. 2: Scheme of QPG operation with two Rydberg atoms. The qubit states $|0\rangle$ and $|1\rangle$ are hyperfine sublevels of the ground state. Atom $a$ is excited to the $n \mathrm{~S}$ state via twophoton absorption, and atom $b$ is excited to the $n \mathrm{P}$ state via one-photon absorption of $\pi / 2$ laser pulses. After dipoledipole interaction they are returned back to the ground state by $3 \pi / 2$ laser pulses.

$|0\rangle$ and $|1\rangle$. Atom $a$ is excited from state $|1\rangle$ to $n \mathrm{~S}$ Rydberg state by a $\pi / 2$ laser pulse via absorption of two photons, while atom $b$ is excited from state $|1\rangle$ to $n \mathrm{P}$ Rydberg state by a $\pi / 2$ laser pulse via one-photon absorption. [Expressions describing $\pi / 2$ pulses for the one- and two-photon transitions will be given below in Eqs. (11), (12), and (13)]. Atoms are returned back to the ground state by the subsequent $3 \pi / 2$ laser pulses. The final phase of the composite wavefunction changes by $\pi$ if the dipole-dipole interaction occurs between exciting and de-exciting laser pulses during time $T$ of Eq.(7). In any other cases of initial states of qubits there will be no phase shifts, as is required for QPG. The laser pulses should be strong and short enough to make excitation and de-excitation insensitive to dipole-induced energy shifts. Eq.(7) determines minimum time of QPG.

We can also analyze an opportunity to observe the frequency shifts of microwave transitions between Rydberg states for modeling the dipole blockade effect [10]. For instance, we may apply a microwave radiation to both atoms initially excited to $n \mathrm{~S}$ state. Recording the absorption spectrum, we should see a shift in the frequency of single-atom excitation of two atoms at the transition $|\mathrm{SS}\rangle \rightarrow|+\rangle$, in comparison with unperturbed frequency of the $n \mathrm{~S} \rightarrow n \mathrm{P}$ transition in a single Rydberg atom. The frequency of the transition $|\mathrm{SS}\rangle \rightarrow|-\rangle$ is also shifted, but probability of this transition is zero due to antisymmetric wave function of $|-\rangle$ state.

Dipole-dipole interaction thus affects the frequencies of transitions in an atomic ensemble. If we apply a pulse of microwave radiation at the unperturbed frequency of the $n \mathrm{~S} \rightarrow n \mathrm{P}$ transition, and the shift is large enough, the final state of Rydberg atom will depend on whether the neighboring atoms are in Rydberg or in ground states. A necessary condition is that the frequency shift $\left(V_{d d} / h\right)$ must exceed spectral width of the pulsed radiation that is ultimately limited by its duration $\tau$ :

$$
V_{d d} / h>>1 / \tau
$$

Fast QC requires $T$ from Eq. (7) or $\tau$ from Eq. (8) to be as short as possible. Eq.(7) is more appropriate to satisfy this requirement, and we see that the QPG gate needs much smaller $V_{d d}$. In order to find $T$ from Eq.(7), we estimate achievable values of $V_{d d}$ for $R \sim 5 \mu \mathrm{m}$ and $n \sim 50$. The radial part $d$ of dipole moments of transitions $(n L \rightarrow n L \pm 1)$ between close Rydberg states is roughly estimated as

$$
d \sim e a_{0} n^{2}
$$

where $e$ is the electron charge. Precise calculations can be made using numerical integration of the Schrödinger's equation described in 26], or with analytical expression and tables derived in [27] for arbitrary effective quantum numbers $n_{e f f}=n-\delta_{L}$ ( $\delta_{L}$ is a quantum defect). As an example, we take the $50 \mathrm{~S}_{1 / 2} \rightarrow 50 \mathrm{P}_{1 / 2}$ microwave transition in $\mathrm{Na}$ at $27.7 \mathrm{GHz}$ and in $\mathrm{Rb}$ at $30.0 \mathrm{GHz}$. Numerically calculated radial part of the dipole moment is 2690 a.u. in $\mathrm{Na}$ and 2550 a.u. in Rb, in good agreement with Eq.(9). With angular part being 1/3, one obtains $\left(V_{d d} / h\right) \sim 10 \mathrm{MHz}$ and $T \sim 50 \mathrm{~ns}$. We see that in principle the QPG operation based on dipole-dipole interaction of Rydberg atoms can be rather fast. However, as we will see below, achievable speed of laser excitation of Rydberg states imposes serious practical limitations.

\section{Laser excitation}

Laser pulses exciting and de-exciting Rydberg states must be much shorter than $T$ to provide sharp switching and ensure the validity of Eq.(6). At $T \sim 50 \mathrm{~ns}$ the pulses should be of $\tau \sim 1-5$ ns duration. The use of short pulses encounters two serious obstacles.

First, the probabilities of single-photon transitions from low-excited to Rydberg states fall as $n^{-3}$ [1]. This is a forcible argument not to use very high Rydberg states: short laser pulses would require too high laser power to generate, e.g., $3 \pi / 2$ pulses for the deexcitation of Rydberg states to ground states. For example, the calculated laser intensity required for a $3 \pi / 2$ pulse of $5 \mathrm{~ns}$ duration is $120 \mathrm{MW} / \mathrm{cm}^{2}$ for the $\left(3 \mathrm{~S}_{1 / 2} \mathrm{~F}=2 \mathrm{M}_{F}=2\right) \rightarrow\left(50 \mathrm{P}_{3 / 2} \mathrm{~F}=3 \mathrm{M}_{F}=3\right)$ transition at $240 \mathrm{~nm}$ in $\mathrm{Na}$, and $30 \mathrm{MW} / \mathrm{cm}^{2}$ for the $\left(5 \mathrm{~S}_{1 / 2} \mathrm{~F}=2\right.$ $\left.\mathrm{M}_{F}=2\right) \rightarrow\left(50 \mathrm{P}_{3 / 2} \quad \mathrm{~F}=3 \quad \mathrm{M}_{F}=3\right)$ transition at $300 \mathrm{~nm}$ in $\mathrm{Rb}$. Even if laser radiation is focused to a spot of $\sim 1 \mu \mathrm{m}$ size, the laser powers should be $\sim 1 \mathrm{~W}$ for $\mathrm{Na}$ and $\sim 0.25$ $\mathrm{W}$ for Rb. Also important is that each atom in the lattice should be controlled by its own laser beam, so the total laser power required for the lattice, say, of 100 atoms would be extremely large. For UV radiation at 250-300 
$\mathrm{nm}$ such powers are difficult to obtain from frequency doubled or tripled cw lasers with external modulators. At the same time, powerful pulsed lasers can not provide the frequency and amplitude stability required for reliable and reproducible quantum operations.

Second, 5 ns pulses have $\sim 200 \mathrm{MHz}$ spectral width that is not enough for spectral resolution of transitions between particular sublevels of the ground and Rydberg states. For example, the calculated fine structure intervals of the $50 \mathrm{P}$ state are $45 \mathrm{MHz}$ in $\mathrm{Na}$ and $819 \mathrm{MHz}$ in $\mathrm{Rb}$. The resolution of $\sim 20 \mathrm{MHz}$ is thus required for excitation of particular sublevels of Rydberg states.

Both of obstacles specified above may be overcome simply by increasing the duration of laser pulses to $\sim 50 \mathrm{~ns}$. However, since time $T$ of dipole-dipole interaction defined by Eq. (77) should be an order of magnitude longer than laser pulses, $T$ has to be increased to $\sim 500 \mathrm{~ns}$ by lowering $\left(V_{d d} / h\right)$ to $\sim 1 \mathrm{MHz}$. The decrease may be achieved by lowering $n$ value to $\approx 30$. Laser power per atom decreases to reasonable values of $\sim 2 \mathrm{~mW}$ for $\mathrm{Na}$ and $\sim 0.5 \mathrm{~mW}$ for $\mathrm{Rb}$, and spectral resolution will be improved to $\sim 20 \mathrm{MHz}$.

In general, Rydberg states with $n=25-50$ in $\mathrm{Na}$ and $\mathrm{Rb}$ atoms may be excited from the ground states by singleor multi-photon absorption. A single-photon process requires UV laser radiation and allows the excitation of $n$ P-states only (in the presence of electric field, however, other states may be excited due to the mixing of the zero-field wavefunctions). For Rb atoms, UV radiation at $300 \mathrm{~nm}$ is required, that may be obtained by doubling the frequency of a Rhodamine $6 \mathrm{G}$ dye-laser at 600 $\mathrm{nm}$. On the other hand, intense $600 \mathrm{~nm}$ radiation itself can excite the $n \mathrm{~S}$ and $n \mathrm{D}$ Rydberg states of Rb via two-photon absorption. The scheme of Fig.2 thus may be realized with two Rhodamine $6 \mathrm{G}$ dye-lasers. These lasers, however, need powerful pumping lasers (Ar or second harmonic of Nd:YAG). For compact inexpensive $\mathrm{Rb}$ experimental setups, it would be better to use multi-step excitation schemes based on frequency stabilized semiconductor lasers, as in 22, 25, 28].

For Na atoms, a two-step scheme $3 \mathrm{~S} \rightarrow 3 \mathrm{P} \rightarrow(n \mathrm{~S}, n \mathrm{D})$ was often used. The first step is excited by the Rhodamine $6 \mathrm{G}$ dye-laser at $589 \mathrm{~nm}$. The second step was usually excited by dye-lasers near $410 \mathrm{~nm}$ pumped by UV nitrogen, eximer, or third harmonic of a Nd:YAG laser. These pumping lasers operate in a pulsed regime at a low repetition rate $(1-100 \mathrm{~Hz})$ that is too slow for QC. Now the $410 \mathrm{~nm}$ dye-laser can be replaced with a second harmonic of cw Ti:sapphire laser or blue semiconductor diode laser.

Finally, taking into account limited speed of laser excitation, we have to specify new optimal parameters for quantum operations: the laser pulses of $\sim 50$ ns duration should be used to excite and de-excite Rydberg atoms at reasonable laser powers; the time of QPG operation should be increased to $\sim 500$ ns by decreasing the principal quantum number to $n \approx 30$.

\section{Degeneracy of atomic states}

We note that model of Fig.2 does not take into account the degeneracy of atomic states. It is important both for the laser excitation and dipole-dipole interaction, since dipole moments of transitions between various magnetic sublevels of degenerate states differ by magnitudes and signs.

Obviously, the ground-state atoms in the optical lattice must be optically pumped to a single magnetic sublevel prior quantum operations. Otherwise, if the ground states are arbitrary mixtures of magnetic sublevels, the speed of laser excitation to Rydberg states will be different for various particular transitions, and $\pi / 2$ laser pulses can not be obtained to transfer all populations to Rydberg states.

Accounting for the magnetic sublevels at the dipoledipole interaction results in several different energy levels of the quasi-molecule of two Rydberg atoms. For instance, there are three energy levels in the case of the simplest microwave transition $n \mathrm{~S}_{1 / 2} \rightarrow n \mathrm{P}_{1 / 2}$ when each state has two magnetic sublevels $M_{J}= \pm 1 / 2$. The behaviour of the quasi-molecule depends now on initial distribution of populations over magnetic sublevels of Rydberg states. Calculations have revealed that Eq.(6) is no more valid, and the phase shift $\pi$ of the wavefunction can not be achieved for degenerate sublevels.

We conclude that degeneracy should be completely lifted for both ground and Rydberg states, e.g., by a homogeneous magnetic field. The field should be strong enough to split the sublevels by a value exceeding the expected energy of dipole-dipole interaction $\left(V_{d d} / h\right) \sim 1$ $\mathrm{MHz}$ in order to ensure validity of Eq. ([6).

\section{E. External fields}

Magnetic field splits degenerate sublevels due to the Zeeman effect. The Zeeman effect in Rydberg atoms and quantum interference of degenerate sublevels at microwave transitions in a weak magnetic field were studied experimentally in our work [29]. The splittings are approximately $2.8 \mathrm{MHz} / \mathrm{Gs}$ for $n \mathrm{~S}_{1 / 2}$ states and 0.93 $\mathrm{MHz} / \mathrm{Gs}$ for $n \mathrm{P}_{1 / 2}$ states. One finds that $\sim 10$ Gs field is required to split the $n \mathrm{P}_{1 / 2}$ state by $10 \mathrm{MHz}$.

Applied magnetic field will also reduce the sensitivity of atoms to stray magnetic fields. For example, the Earth's magnetic field of the 0.5 Gs magnitude alone splits atomic states by $\sim 0.5 \mathrm{MHz}$. This results in a phase difference of the order of $\pi$ obtained by magnetic sublevels during $\sim 1 \mu \mathrm{s}$ interaction time, and in a mixing of populations due to the precession of magnetic moment. The phase is important when optical or microwave transitions are excited between coherently prepared degenerate states that can interfere. Our previous experiment on microwave Hanle effect at the $37 \mathrm{P}_{3 / 2} \rightarrow 37 \mathrm{~S}_{1 / 2}$ microwave transition in $\mathrm{Na}$ atoms demonstrated variation of the probability by $50 \%$ when magnetic field was varied 


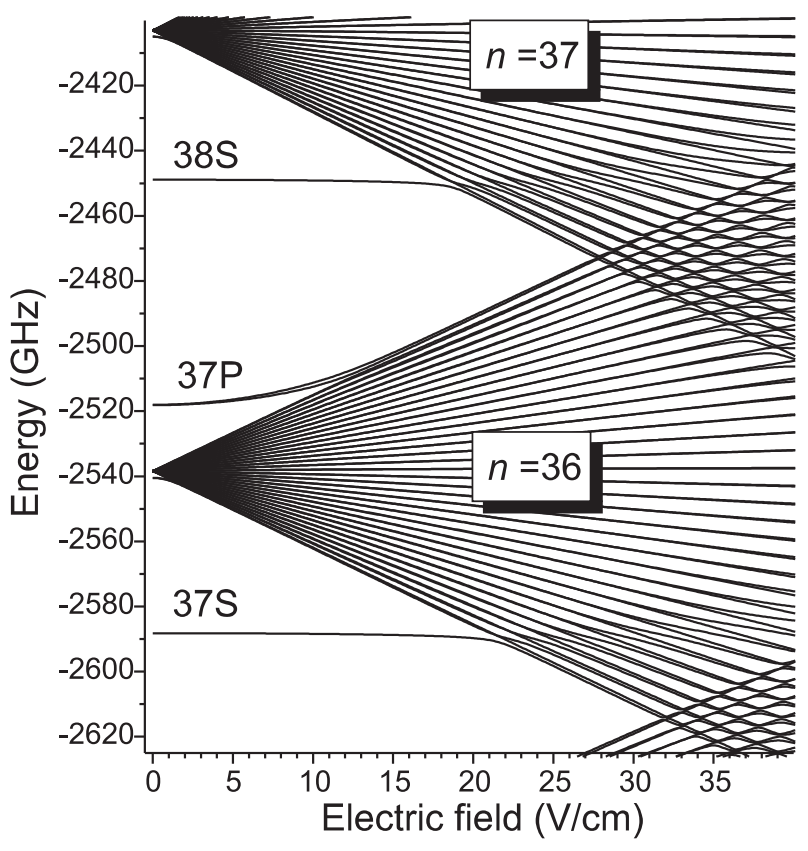

FIG. 3: Numerically calculated Stark diagram of the sodium Rydberg states near $n=36$.

within 20 mGs around zero [29]. In another experiment with this transition we observed a mixing of populations due to the precession 30. Application of a magnetic field of $\sim 10$ Gs will define the quantization axis and suppress the precessions from stray fields, so that no additional magnetic screening will be needed.

Electric field influences on Rydberg atoms especially strongly. In Fig. 3 a part of the numerically calculated Stark diagram for the sodium Rydberg states near $n=36$ is shown (the calculations have been made using the diagonalization procedure described in [26]). The electric field of several $\mathrm{V} / \mathrm{cm}$ significantly changes energies of all Rydberg levels. The hydrogen-like states with $L>1$ experience linear Stark effect and become completely mixed by the electric field, while non-hydrogenic $n \mathrm{~S}$ and $n \mathrm{P}$ states have large quantum defects in sodium (1.348 and 0.855 correspondingly) and experience quadratic Stark effect up to the field of $10 \mathrm{~V} / \mathrm{cm}$. The mixing of highmoment states makes them inapplicable to QC since any stray electric field would redistribute populations. We conclude that in $\mathrm{Na}$ only $\mathrm{S}$ and $\mathrm{P}$ states would be suitable to $\mathrm{QC}$ because they are stable against electric field fluctuations. An exception is the D-state (0.015 quantum defect) that also has quadratic Stark effect in the electric fields below $0.1 \mathrm{~V} / \mathrm{cm}$, and is not mixed with other states in such fields.

Usage of $\mathrm{S}$ and $\mathrm{P}$ states has an advantage of ability to use Stark-switching technique to control the interaction with $\mathrm{cw}$ radiation in a coherent way, as it was demonstrated in our recent experiment on Stark-switched Ramsey interferometry of the $37 \mathrm{~S}_{1 / 2} \rightarrow 37 \mathrm{P}_{1 / 2}$ transition 31]. The Stark-switching can significantly simplify experiments because it eliminates the need to use fast mod- ulators for $\mathrm{cw}$ radiation, but it would require phase corrections of quantum operations to account for additional phase shifts from the Stark effect. Lower Rydberg states with $n \approx 30$ are less sensitive to the electric field than states in Fig. 3 since polarizabilities scale as $n_{e f f}^{7}$, and no electric field screening will be required if $30 \mathrm{~S}$ and $30 \mathrm{P}$ states will be used.

Blackbody radiation (BBR) induces the microwave transitions to nearby Rydberg states and shortens their lifetimes. In order to decrease its influence, experiments with Rydberg atoms are usually made with thermal shields cooled to liquid nitrogen $(77 \mathrm{~K})$ or liquid helium $(4 \mathrm{~K})$ temperatures. This technique requires vacuum cryostats and nontransparent copper shields that would complicate experimental arrangements and optical access to individual atoms in the optical lattice. We have to decide is this cooling needed in the experiments on QC. Without the BBR, the radiative lifetimes scale as $n_{\text {eff }}^{3}$ for the states with $L \ll n[32$. In a sodium atom $\tau(30 \mathrm{~S}) \approx 30 \mu \mathrm{s}$ and $\tau(30 \mathrm{P}) \approx 300 \mu \mathrm{s}$. With the BBR at a room temperature $300 \mathrm{~K}$, effective lifetimes decrease to $\tau(30 \mathrm{~S}) \approx 20 \mu \mathrm{s}$ and $\tau(30 \mathrm{P}) \approx 30 \mu \mathrm{s}$. Although the decrease is significant, we see that influence of the BBR is of small importance if the operation time is $\sim 500 \mathrm{~ns}$, and no cooled environment is needed. However, one should keep in mind that if experiments are made with a detection system that uses some closed metallic surrounding, enhancement of interaction of atoms with the BBR may occur since the surrounding works as a microwave cavity increasing spectral density of the BBR field [23].

\section{F. Detection}

Sensitive detection of single Rydberg atoms is a crucial point of the future experiments on QC. Only technique of the Selective Field Ionization (SFI) 11] is applicable to high Rydberg states with $n>20$ since spontaneous fluorescence is extremely low. A Rydberg atom in the $n L$ state can ionize with a probability close to 1 by an electric field whose critical strength $E_{c}$ strongly depends on the effective quantum number according to the formula

$$
E_{c} \approx 3.2 \cdot 10^{8} n_{\text {eff }}^{-4} \quad \mathrm{~V} / \mathrm{cm} .
$$

For $n \approx 30$ one finds that $E_{c} \approx 400 \mathrm{~V} / \mathrm{cm}$ is required.

Single electrons resulted from ionization are usually detected by channeltron or channel-plate detectors with a probability limited mostly by the transparency of the input electric field grid (typically 50-80 \%). The SFI also allows to measure distribution of populations over different Rydberg states, if a ramp of electric field is applied.

Obvious drawback of this method is the destruction of Rydberg atoms in the ionization process. Hence, it cannot be applied to continuous control of Rydberg atoms in $\mathrm{QC}$, and may be used only for the preliminary tunings before quantum computation. After loading of optical lattice with single atoms, all intermediate operations 
must be performed without the SFI detection, and only final measurements can be made with it.

Individual SFI detection of single Rydberg atoms in the optical lattice is another technical problem. Since atoms in the lattice will be spaced by $\sim 5 \mu \mathrm{m}$, position sensitive channel-plate detectors must have proper spatial resolution to ensure detection of each atom. Also, if individual detection of atoms is needed, each atom should be provided with its own electric field electrode that does not affect other atoms. Such configuration will be difficult to realize in practice, and the only way will be use of the common electric field plate for the whole lattice. This means that preliminary tunings can be made only for the whole atomic ensemble.

\section{G. Summary for experimental limitations}

- Minimum distance $R$ between atoms in optical lattice has to be $5 \mu \mathrm{m}$ to provide free optical access to each atom. This distance is compatible with conservative quasi-electrostatic $\mathrm{CO}_{2}$-laser traps having extremely low scattering rate of photons.

- At this $R$, principal quantum numbers $n$ of Rydberg states should not exceed 70 to avoid dephasing collisions between neighboring Rydberg atoms.

- With $R \approx 5 \mu \mathrm{m}$ and $n \approx 50$, maximum energy shifts $V_{d d}$ of Rydberg states due to the dipole-dipole interaction may reach $10 \mathrm{MHz}$, and minimum time required to perform a QPG operation is $50 \mathrm{~ns}$. But in this case exciting laser pulses should be of $\sim 5$ ns duration and $\sim 1$ $\mathrm{W}$ power, even if focused to a spot of $\sim 1 \mu \mathrm{m}$ size.

- In order to use reasonable laser powers of $\sim 1 \mathrm{~mW}$ per atom and improve spectral resolution to $\sim 20 \mathrm{MHz}$ at excitation and de-excitation of Rydberg states, duration of the laser pulses should be increased to 50 ns. The time of dipole-dipole interaction should be also increased to $\sim 500$ ns by lowering $n$ to 30 .

- Magnetic field of $\sim 10$ Gs has to be applied to lift the degeneracy of ground and Rydberg states, to provide selective excitation of particular magnetic sublevels and to suppress the precession of magnetic moment in stray magnetic fields. Ground-state atoms should be optically pumped to a certain magnetic sublevel prior quantum computations.

- Stray electric fields should be suppressed to avoid mixing of Rydberg states, or low-moment states having quadratic Stark effect should be chosen, for example, $n \mathrm{~S}$ and $n \mathrm{P}$ states.

- Suppression of the blackbody radiation at $300 \mathrm{~K}$ is not required if the time of the dipole-dipole interaction is $\sim 500 \mathrm{~ns}$.

- The SFI detection of Rydberg atoms may be applied only before quantum computations for preliminary tunings and afterwards for final measurements.

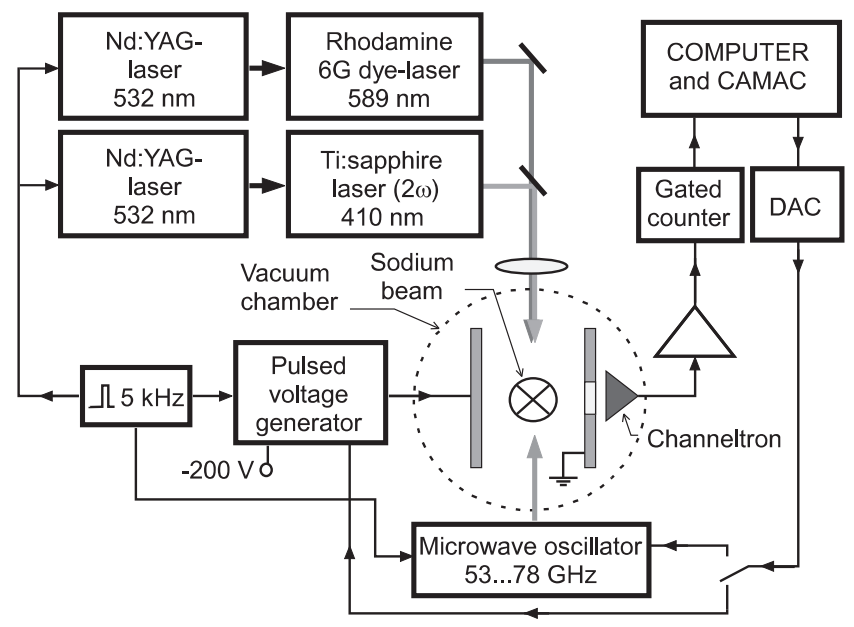

FIG. 4: Experimental setup.

\section{MICROWAVE SPECTROSCOPY OF MULTI-ATOM EXCITATIONS}

QC based on cold atoms in optical lattices may be realized, in principle, in the nearest future. The above considerations impose the limitations that require additional experimental tests. Some experiments can be made with atoms in a thermal atomic beam. For example, important for the dipole blockade [10] spectra of multi-atom excitations of Rydberg atoms have not been reported yet. Even without dipole-dipole interaction, these spectra are expected to be different from that of single-atom excitations, since various final states of the ensemble of Rydberg atoms may be selectively detected with the SFI technique. We studied these spectra at microwave transitions in $\mathrm{Na}$ Rydberg atoms in an effusive atomic beam.

\section{A. Experimental setup}

The sodium atoms were excited to an initial $37 \mathrm{~S}_{1 / 2} \mathrm{Ry}-$ dberg state by a two-step scheme $3 \mathrm{~S}_{1 / 2} \rightarrow 3 \mathrm{P}_{3 / 2} \rightarrow 37 \mathrm{~S}_{1 / 2}$ with two pulsed tunable lasers (Rhodamine $6 \mathrm{G}$ and the second harmonic of Ti:sapphire) at a $5-\mathrm{kHz}$ repetition rate (Fig.4). Laser pulses were synchronized and acted simultaneously, exciting several Rydberg atoms per pulse on average.

Experiments were carried out in a vacuum chamber with a sodium effusive atomic beam at 450-K temperature and $10^{8} \mathrm{~cm}^{-3}$ atom density in a $5 \cdot 10^{-5} \mathrm{~cm}^{3}$ excitation volume. No collisions and dipole-dipole interaction were expected in such conditions since the average distance between ground-state atoms was $\sim 10 \mu \mathrm{m}$, and the average distance between Rydberg atoms was $\sim 100 \mu \mathrm{m}$. The laser radiation was focused perpendicularly to the beam, so that the point of laser excitation was localized within $0.2 \mathrm{~mm}$ along the beam. The Rydberg atoms were passed between the two copper plates separated by $8.15 \mathrm{~mm}$. A pulsed voltage was applied to one of them, 
to form a homogeneous electric field for the detection of atomic populations by the SFI. The other plate had a 9-mm-diameter hole, covered by a grid of $80 \%$ optical transparency, through which the electrons appearing in the SFI were guided to an input window of a standard channeltron VEU-6. The excitation region was surrounded by copper shields, and all parts of the detection system were cooled to a liquid-nitrogen temperature 77 $\mathrm{K}$ to decrease the influence of the blackbody radiation. The laboratory magnetic field was compensated for below $5 \mathrm{mGs}$ in all directions by three pairs of Helmholtz coils.

A microwave radiation tunable in the $53-78 \mathrm{GHz}$ range was introduced to the excitation region through a hole in a waveguide connected to a standard backward-tube oscillator G4-142. It was locked to a quartz synthesizer and operated in cw mode with a linewidth below $10 \mathrm{kHz}$. The spectra of microwave transitions were recorded by scanning the frequency of the synthesizer. Spatial distribution of the microwave field was rather complex since it was formed as arbitrary standing wave after multiply reflections from surrounding copper surfaces that may be considered as a low-Q $(\mathrm{Q} \sim 100)$ microwave cavity. The point of laser excitation was adjusted to a maximum of the standing wave following the procedure described in our previous work [29].

The time evolution of the signals was as follows. At time $t=0$ a laser pulse of 50 ns duration populated the $37 \mathrm{~S}_{1 / 2}$ state. Microwave radiation tuned near the frequency of the one-photon $37 \mathrm{~S}_{1 / 2} \rightarrow 37 \mathrm{P}_{1 / 2}$ or two-photon $37 \mathrm{~S}_{1 / 2} \rightarrow 38 \mathrm{~S}_{1 / 2}$ transition was applied continuously. It populated the final $37 \mathrm{P}_{1 / 2}$ or $38 \mathrm{~S}_{1 / 2}$ state. The interaction of atoms with radiation occurred until $2.8 \mu \mathrm{s}$, at which moment a ramp of strong electric field with a risetime of $2 \mu \mathrm{s}$ was applied for the SFI detection. The output pulses of the channeltron appeared at 3.5-4.5 $\mu \mathrm{s}$ delay relative to the laser pulse when the field reached critical values for the different states. Final $37 \mathrm{P}_{1 / 2}$ and $38 \mathrm{~S}_{1 / 2}$ states had equal critical fields for the SFI, since in a strong electric field their energies coincide, as can be seen from the Stark map in Fig.3.

The SFI technique allows for detection of single Rydberg atoms with probability close to 1 if electrons are detected by the channeltron (our channeltron had typical gain about $10^{8}$ ). A total charge of the output pulses of the channeltron was integrated separately for the lower and upper states of transitions for each laser pulse, and measured by fast Analog-to-Digital Converter (ADC). After amplification, single-electron pulses had $400 \mathrm{mV}$ average amplitudes that fluctuated within $350-450 \mathrm{mV}$ from pulse to pulse with a gaussian distribution of amplitudes. Two-electron pulses had $800 \mathrm{mV}$ average amplitudes and fluctuated within 700-900 mV. Tree-electron pulses were peaked at $1200 \mathrm{mV}$, etc. Selective determination of the total number of Rydberg atoms was achieved up to five atoms, after which value the multi-electron signals started to overlap. We thus were able to distinguish up to 5 atoms and study the spectra of multi-atom

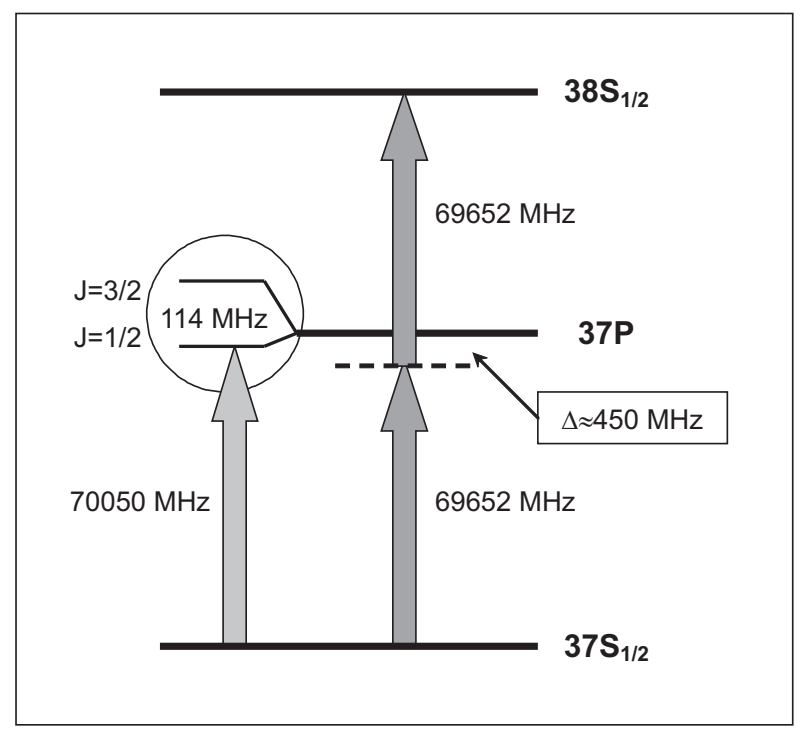

FIG. 5: Scheme of the one-photon $37 \mathrm{~S}_{1 / 2} \rightarrow 37 \mathrm{P}_{1 / 2}$ and twophoton $37 \mathrm{~S}_{1 / 2} \rightarrow 38 \mathrm{~S}_{1 / 2}$ transitions in sodium Rydberg atoms.

excitations at microwave transitions.

\section{B. Single-atom spectra}

The schemes of the one-photon $37 \mathrm{~S}_{1 / 2} \rightarrow 37 \mathrm{P}_{1 / 2}$ and two-photon $37 \mathrm{~S}_{1 / 2} \rightarrow 38 \mathrm{~S}_{1 / 2}$ microwave transitions in $\mathrm{Na}$ atoms are shown in Fig.5. The relevant effective lifetimes are about $60 \mu \mathrm{s}$ for the S-states and $150 \mu \mathrm{s}$ for the P-state, so that spontaneous decay may be neglected during the interaction time $\tau=2.8 \mu \mathrm{s}$. In this approximation, single-atom probabilities of transitions are calculated within the two- and three-level models in a rotatingwave approximation for a square microwave pulse. The evolution of populations of the upper state $37 \mathrm{P}_{1 / 2}$ of the one-photon transition and $38 \mathrm{~S}_{1 / 2}$ of the two-photon transition is described by the following formulas:

$$
\begin{gathered}
\rho_{37 P}=\frac{\Omega_{1}^{2}}{\delta^{2}+\Omega_{1}^{2}} \sin ^{2}\left(\frac{\tau}{2} \sqrt{\delta^{2}+\Omega_{1}^{2}}\right), \\
\rho_{38 S}=\frac{\Omega_{2}^{2}}{\left(\delta-\delta_{0}\right)^{2}+\Omega_{2}^{2}} \sin ^{2}\left(\tau \sqrt{\left(\delta-\delta_{0}\right)^{2}+\Omega_{2}^{2}}\right) .
\end{gathered}
$$

Here $\Omega_{1}=\left(d_{1} E / \hbar\right)$ is a Rabi frequency of the $37 \mathrm{~S}_{1 / 2} \rightarrow 37 \mathrm{P}_{1 / 2}$ transition $\left(d_{1}\right.$ is a matrix element of the dipole moment, $E$ is the strength of the microwave field), $\delta$ is a detuning from exact resonance; $\Omega_{2}=d_{1} d_{2} E^{2} /\left(4 \hbar^{2} \Delta\right)$ is a Rabi frequency of the $37 \mathrm{~S}_{1 / 2} \rightarrow 38 \mathrm{~S}_{1 / 2}$ transition $\left(d_{1}, d_{2}\right.$ are matrix elements of the dipole moment for the $37 \mathrm{~S} \rightarrow 37 \mathrm{P}$ and $37 \mathrm{P} \rightarrow 37 \mathrm{~S}$ intermediate transitions, $\Delta$ is a detuning of the virtual intermediate level of the two-photon transition from the 

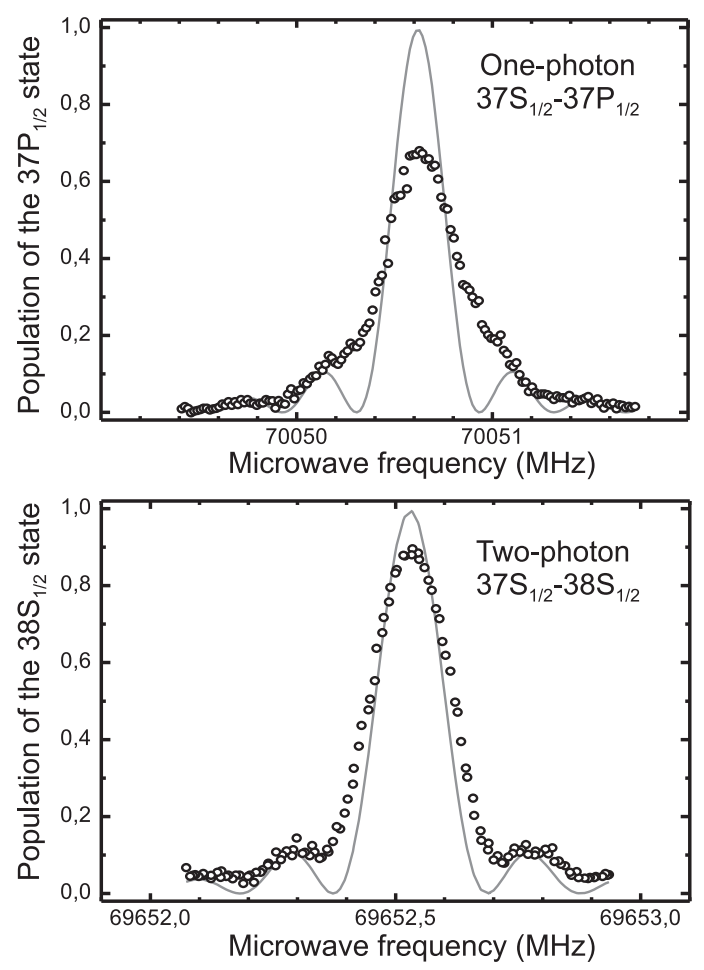

FIG. 6: Comparison of experimental records of the spectrum of the one-photon $37 \mathrm{~S}_{1 / 2} \rightarrow 37 \mathrm{P}_{1 / 2}$ transition (upper picture) and two-photon $37 \mathrm{~S}_{1 / 2} \rightarrow 38 \mathrm{~S}_{1 / 2}$ transition (lower picture) with theoretical calculations (solid lines).

real 37P level), and $\delta_{0}=\left(d_{1}^{2}-d_{2}^{2}\right) E^{2} /\left(8 \hbar^{2} \Delta\right)$ is a power shift of the two-photon transition.

The experimental spectra obtained for the one- and two-photon transitions are compared in Fig.6. The solid lines are theoretical curves from Eqs.(11) and (12). In this experiment we attempted to realize a NOT operation (inversion) in the center of atomic line. The microwave intensity was adjusted to achieve maximum population of the upper state in the center. It is seen from Fig.6 that in the case of one-photon transition population of the $37 \mathrm{P}_{1 / 2}$ state does not exceed $70 \%$, and the shape of the resonance substantially differs from the theoretical one. The shape is closer to Lorentzian rather than to that predicted by Eq. (11). In the case of two-photon transition, population of the upper $38 \mathrm{~S}_{1 / 2}$ state reaches $90 \%$, and the shape of the resonance better agrees with theory. There are two reasons for distortion of the experimental single-atom spectra.

First, atoms in the atomic beam have a Maxwell velocity distribution with average velocity $600 \mathrm{~m} / \mathrm{s}$. This value corresponds to $150 \mathrm{kHz}$ Doppler broadening of the one-photon microwave transitions at $70 \mathrm{GHz}$. The broadening is less important for two-photon transitions, since photons in the standing wave may be absorbed from two traveling waves propagating in opposite direction, and the Doppler effect is partly compensated for. More important is that effective time of interaction depends on the atom velocity since atoms are passed through the nodes and antinodes of the standing wave, so that maxima and minima in the spectra may wash out.

Second, the spectra in Fig.6 were recorded in identical experimental conditions except the difference in the microwave intensity. We believe that better agreement with theory for the two-photon transition, and disagreement for the one-photon transition originate also from significant difference in the microwave photon density required for the NOT operation. According to Eqs. (111) and (12), inversion is achieved with $\pi / 2$ microwave pulses:

$$
\left\{\begin{array}{l}
\Omega_{1} \tau / 2=\pi / 2 \\
\Omega_{2} \tau=\pi / 2
\end{array}\right.
$$

These relations allow the calculation of $E$ value, provided relevant dipole moments are known. Their radial parts were calculated numerically following to Ref. [26], and found to be 1460 a.u. for the $37 \mathrm{~S} \rightarrow 37 \mathrm{P}$ transition and 1430 a.u. for the $37 \mathrm{P} \rightarrow 38 \mathrm{~S}$ transition. Angular parts were taken for the transitions induced by linearly polarized microwave field, with the fine-structure taken into account.

The average density of microwave photons at the antinode of a standing wave was estimated from the following energy relation: volume density of the energy of the electromagnetic field is

$$
\frac{d W}{d V}=\frac{\varepsilon_{0} E^{2}}{2}
$$

where $\varepsilon_{0}$ is a dielectric constant. Dividing this expression by $\hbar \omega$, we obtain a volume density of photons $d N / d V$ about $50 \mathrm{~cm}^{-3}$ for the one-photon $37 \mathrm{~S}_{1 / 2} \rightarrow 37 \mathrm{P}_{1 / 2}$ transition, and about $10^{5} \mathrm{~cm}^{-3}$ for the two-photon $37 \mathrm{~S}_{1 / 2} \rightarrow 38 \mathrm{~S}_{1 / 2}$ transition.

Extremely low number of photons at the one-photon transition requires huge attenuation of the output power of the microwave oscillator. Even with such attenuation, we noticed that photons leaking the oscillator through its case penetrated into the interaction region through the holes in the detection system and interfered with photons coming from the waveguide. This interference caused instability in the spatial distribution of the microwave field and also resulted in fluctuations of effective interaction time. To some extent, this assumption was confirmed experimentally when we decreased the time of interaction to $0.3 \mu \mathrm{s}$, and increased the microwave intensity in the waveguide by three times. The one-photon resonance became wider, but in the center of the line we were able to transfer about $80 \%$ of population to the upper state, and better agreement of the spectrum with Eq.(11) was found.

Obviously, the Doppler effect will be suppressed to a high degree in the case of cold atoms trapped in the op- 


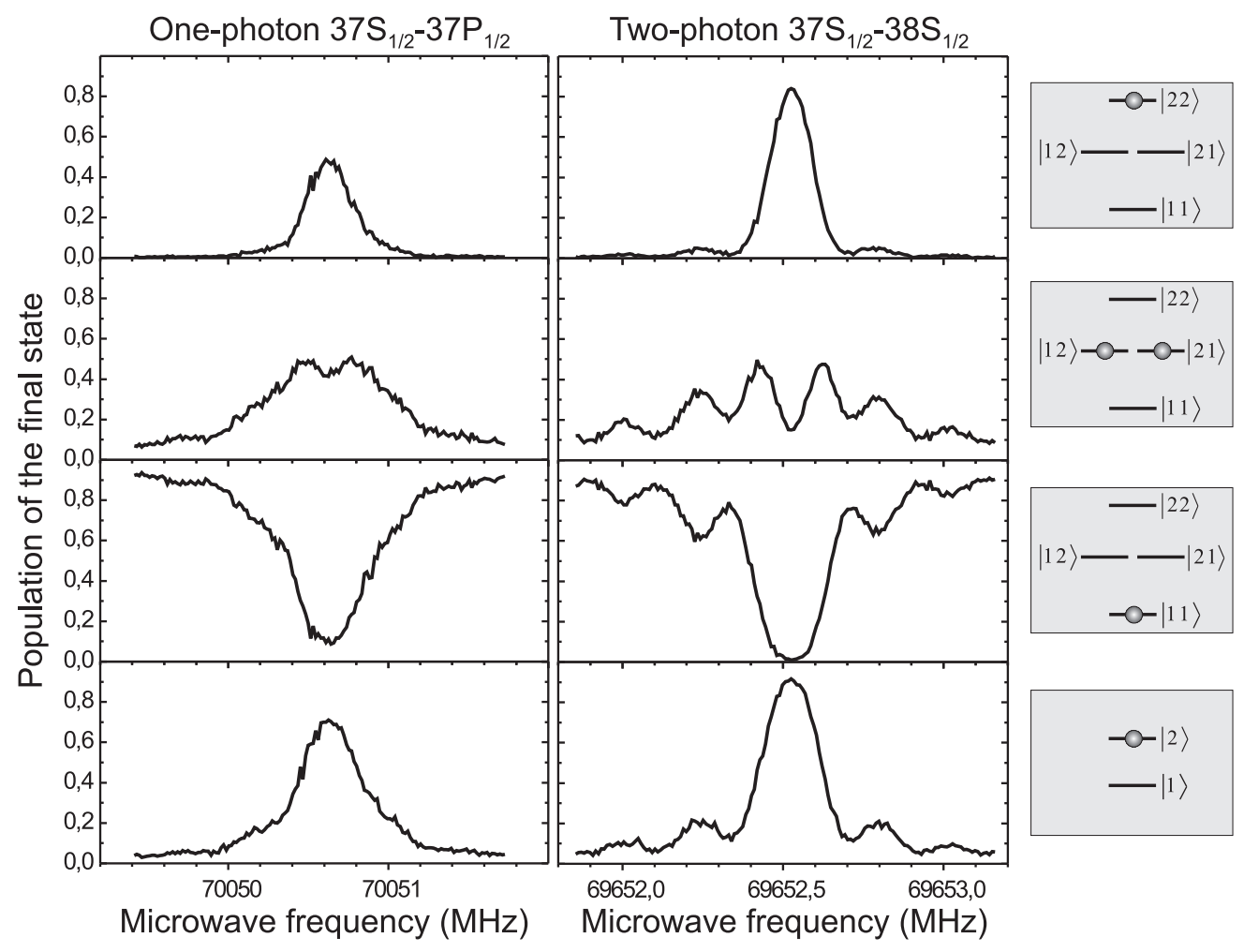

FIG. 7: Spectra of single-atom (lower trace) and two-atom (upper traces) excitations of the one-photon $37 \mathrm{~S}_{1 / 2} \rightarrow 37 \mathrm{P}_{1 / 2}$ and two-photon $37 \mathrm{~S}_{1 / 2} \rightarrow 38 \mathrm{~S}_{1 / 2}$ transitions. The right-hand schemes indicate what final states of a composite system are detected. State 1 is the initial $37 \mathrm{~S}_{1 / 2}$ state. State 2 is the final $37 \mathrm{P}_{1 / 2}$ state (for the one-photon transition) or $38 \mathrm{~S}_{1 / 2}$ state (for the two-photon transition).

tical lattices, but vibrational motion of atoms and fluctuations of laser intensity and frequency may affect the precision of NOT operations in a way similar to that in Fig.6.

\section{Multi-atom spectra}

Our detection system measured the total number of Rydberg atoms and distribution of populations after each laser pulse. We accumulated multi-atom signals for a short time (1-2 s) and automatically sorted them by number of detected atoms and by distribution of populations. No dipole-dipole interaction was observed in this experiment since average distance between Rydberg atoms was $\sim 100 \mu \mathrm{m}$. The experiments were made with of onephoton $37 \mathrm{~S}_{1 / 2} \rightarrow 37 \mathrm{P}_{1 / 2}$ and two-photon $37 \mathrm{~S}_{1 / 2} \rightarrow 38 \mathrm{~S}_{1 / 2}$ transitions. We denote the initial $37 \mathrm{~S}_{1 / 2}$ state to be state 1 , and the final states $37 \mathrm{P}_{1 / 2}$ or $38 \mathrm{~S}_{1 / 2}$ to be state 2 .

In the simplest case of two-atom excitations, an initial state is $|11\rangle$, while four final states of two atoms may be detected after interaction with the microwave field: $|11\rangle$, $|12\rangle,|21\rangle$, and $|22\rangle$. Direct products of single-atom wavefunctions describe these states if atoms do not interact with each other. The probabilities to find two atoms af- ter a measurement in a particular state may be expressed as

$$
\left\{\begin{array}{l}
\rho_{|11\rangle}=\rho_{37 S} \times \rho_{37 S} \\
\rho_{|12\rangle}=\rho_{|21\rangle}=\rho_{37 S} \times \rho_{37 P} \\
\rho_{|22\rangle}=\rho_{37 P} \times \rho_{37 P}
\end{array}\right.
$$

for the one-photon transition, and

$$
\left\{\begin{array}{l}
\rho_{|11\rangle}=\rho_{37 S} \times \rho_{37 S} \\
\rho_{|12\rangle}=\rho_{|21\rangle}=\rho_{37 S} \times \rho_{38 S} \\
\rho_{|22\rangle}=\rho_{38 S} \times \rho_{38 S}
\end{array}\right.
$$

for the two-photon transition. Here $\rho_{37 P}$ and $\rho_{38 S}$ are the probabilities to find single atom in a final state given by Eqs. (11) and (12), and $\rho_{37 S}=\left(1-\rho_{37 P}\right)$ or $\rho_{37 S}=\left(1-\rho_{38 S}\right)$ are probabilities of the single atom to remain in the $37 \mathrm{~S}$ state.

In Fig.7 we present the experimental records of the two-atom spectra (three upper traces), and compare them with a single-atom spectrum (lower trace). Microwave intensity was adjusted to provide maximum population transfer to the upper state. The right-hand schemes show what final states of the composite system 
of two atoms were detected. The comparison of the spectra with calculations by Eq.(16) revealed good agreement for the two-photon transition, confirming that we indeed detected only two atoms. Agreement was much worse for the one-photon transition due to the reasons discussed in the previous subsection.

All two-atom spectra are different from that of a single atom. We note two important features: (i) probability of excitation of only one of two atoms has a narrow dip in the line center due to large probability to excite both atoms, and (ii) probability of excitation of both atoms has narrower spectrum than single-atom spectrum. This may have applications for high-resolution spectroscopy as a method of narrowing of atomic lines. For example, in the case of $N$ Rydberg atoms the probability to transfer all atoms to the upper state 2 is

$$
\rho_{|222 \ldots \ldots 2\rangle}=\left(\rho_{38 S}\right)^{N}
$$

for the two-photon transition. If this final state is selectively detected in the atomic ensemble, as in our experiment, observed spectrum of full excitation will be much narrower than single atom spectrum. The width of multi-atom resonances may be estimated by substituting the Lorentzian pre-factor of Eq.(12) to Eq.(17). The full width at half maximum is

$$
\Delta \omega_{N}=2 \Omega_{2} \sqrt{\sqrt[N]{2}-1} .
$$

In the limit of $N \gg 1$, one finds the relationship between the widths of the single-atom and multi-atom resonances:

$$
\frac{\Delta \omega_{1}}{\Delta \omega_{N}} \approx \sqrt{\frac{N}{\ln 2}}
$$

For example, the resonance becomes 2.7 times narrower with $N=5$.

In Fig. 8 the spectra of full multi-atom excitations observed at the two-photon transition are presented. The spectra of full excitations for 1,3 , and 5 atoms display that multi-atom resonances are indeed much narrower than single-atom ones, but their amplitudes become smaller with growing $N$. We conclude that ultimate resolution of this method of narrowing of atomic lines is limited by the ability to distinguish full excitation of the atomic ensemble from the partial one, and by the ability to transfer populations of all atoms to the upper states, otherwise amplitude of the multi-atom resonance would rapidly decrease with growing $N$.

Although our experiments were made with atomic beam and no dipole-dipole interaction was observed, obtained spectra revealed the main features of single- and multi-atom excitations. We expect that similar spectra would be observed, e.g., at the laser excitation of an ensemble of Rydberg atoms from the ground state.

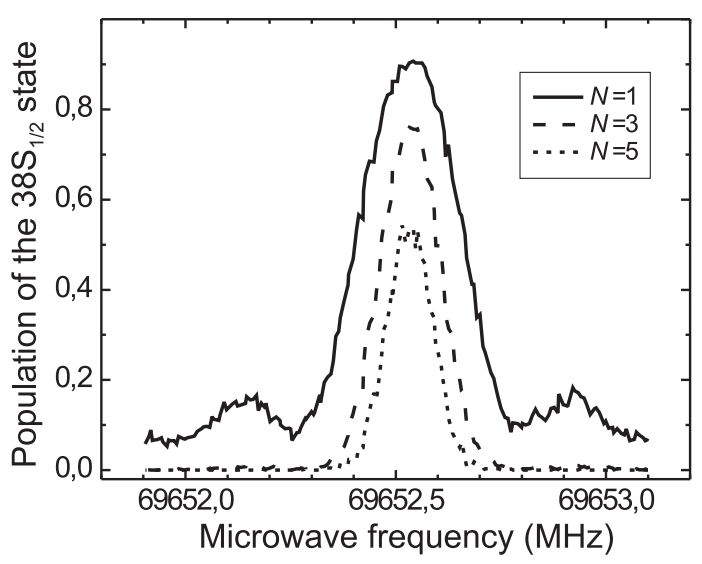

FIG. 8: Spectra of full multi-atom excitations of the twophoton transition $37 \mathrm{~S}_{1 / 2} \rightarrow 38 \mathrm{~S}_{1 / 2}$ for various number $N$ of Rydberg atoms.

Now we can also understand how the dipole-dipole interaction may affect the spectra. In the case of two Rydberg atoms and one-photon $n \mathrm{~S}_{1 / 2} \rightarrow n \mathrm{P}_{1 / 2}$ microwave transition, it will change the frequencies of transitions from the initial $|\mathrm{SS}\rangle$ state to the strongly interacting $|\mathrm{SP}\rangle$ and $|\mathrm{PS}\rangle$ states, while the frequency of the transition to $|\mathrm{PP}\rangle$ state will be unaffected but its probability will decrease. An experiment on the observation of the dipoledipole interaction between few close Rydberg atoms excited in extremely small volume of a dense atomic beam is in progress now.

\section{SUMMARY}

We have analyzed the applicability of Rydberg atoms to quantum computers and defined the parameters of Rydberg atoms and experimental arrangements that are most suitable to realize a quantum phase gate in optical lattices of cold alkali atoms. The principal quantum number $n \sim 30$, low orbital moments $(L \sim 0,1,2)$, $\sim 5 \mu \mathrm{m}$ interatomic distance, $50 \mathrm{~ns}$ exciting laser pulses, and $\sim 500 \mathrm{~ns}$ time of dipole-dipole interaction seem to be optimal. Modeling experiments on microwave spectroscopy of a few Na Rydberg atoms in the atomic beam revealed some important features in the spectra of singleand multi-atom excitation for the one- and two-photon transitions. The quantum NOT operation with single atoms was found to be affected by the Doppler effect and fluctuations of the microwave field. The spectrum of complete excitation of several Rydberg atoms was much narrower than that of single atom. This effect may be applied to high-resolution spectroscopy for the narrowing of atomic lines. Similar spectra of single- and multi-atom excitations will be observed at the laser excitation of an 
ensemble of ground-state atoms to Rydberg states.

\section{Acknowledgments}

This work was supported by the Russian Foundation for Basic Research, Grant Nos. 02-02-16323, 03-02-
06867, and by INTAS, Grant No.2001-155.
[1] D. P. DiVincenzo, Science 270, 255 (1995); J. Gruska, Quantum Computing (McGraw-Hill, London, 1999); A. M. Steane, Rep. Prog. Phys. 61, 117(1998).

[2] C. H. Bennett and D. P. DiVincenzo, Nature (London) 404, 247 (2000).

[3] H. J. Metcalf and P. van der Straten, Laser Cooling and Trapping (Springer, New York, 1999); R. Grimm, M. Weidemüller, and Yu. B. Ovchinnikov, Adv. At. Mol. Opt. Phys. 42, 95 (2000).

[4] R. Dumke, M. Volk, T. Muther, F. B. J. Buchkremer, G. Birkl, and W. Ertmer, Phys. Rev. Lett. 89, 097903 (2002).

[5] N. Schlosser, G. Reymond, I. Protsenko, and P. Granier, Nature (London) 411, 1024 (2001).

[6] N. Schlosser, G. Reymond, and P. Grangier, Phys. Rev. Lett. 89, 023005 (2002).

[7] R. P. Feynman, Opt. News 11, 11 (1985).

[8] G. K. Brennen, C. M. Caves, P. S. Jessen, and I. H. Deutsch, Phys. Rev. Lett. 82, 1060 (1999); G. K. Brennen, I. H. Deutsch, and P. S. Jessen, Phys. Rev. A 61, 062309 (2000).

[9] D. Jaksch, J. I. Cirac, P. Zoller, S. L. Rolston, R. Côté, and M. D. Lukin, Phys. Rev. Lett. 85, 2208 (2000).

[10] M. D. Lukin, M. Fleischhauer, R. Côté, L. M. Duan, D. Jaksch, J. I. Cirac, and P. Zoller, Phys. Rev. Lett. 87, 037901 (2001).

[11] Rydberg States of Atoms and Molecules, ed. by R. F. Stebbings and F. B. Dunning (Cambridge University Press, Cambridge, 1983); T. F. Gallagher, Rydberg Atoms (Cambridge University Press, Cambridge, 1994).

[12] I. E. Protsenko, G. Reymond, N. Schlosser, and P. Grangier, Phys. Rev. A 65, 052301 (2002).

[13] M. S. Safronova, C. J. Williams, and C. W. Clark, Phys. Rev. A 67, 040303(R) (2003).

[14] R. G. Unanyan and M. Fleischhauer, Phys. Rev. A 66, 032109 (2002).

[15] C. Boisseau , I. Simboten and R. Côté, Phys. Rev. Lett. 88, 133004 (2002); S. M. Farooqi, D. Tong, S. Krishnan, J. Stanojevic, Y. P. Zhang, J. R. Ensher, A. S. Estrin, C. Boisseau, R. Côté, E. E. Eyler, and P. L. Gould, Phys. Rev. Lett. 91, 183002 (2003).

[16] J. M. Raimond, G. Vitrant, and S. Haroche, J. Phys. B
14, L655 (1981).

[17] D. Tong, S. M. Farooqi, J. Stanojevic, S. Krishnan, Y. P. Zhang, R. Côté, E. E. Eyler, and P. L. Gould, arXiv:physics/0402113 (2004); K. Singer, M. Reetz-Lamour, T. Amthor, L. G. Marcassa, and M. Weidemüller, arXiv:physics/0404075 (2004).

[18] W. R. Anderson, J. R. Veale, and T. F. Gallagher, Phys. Rev. Lett. 80, 249 (1998); I. Mourachko, D. Comparat, F. de Tomasi, A. Fioretti, P. Nosbaum, V. M. Akulin, and P. Pillet, Phys. Rev. Lett. 80, 253 (1998).

[19] K. Afrousheh, Z. Bohlouli, D. Vagale, A. Mugford, M. Fedorov, and J. D. D. Martin, Submitted to Phys. Rev. Lett. (2004).

[20] H. A. Bethe and E. E. Salpeter, Quantum Mechanics of One- and Two-Electron Atoms (Springer, Berlin, 1957).

[21] J. Neukammer, H. Rinneberg, K. Vietzke, A. Konig, H. Hieronymus, M. Kohl, H. -J. Grabka, and G. Wunner, Phys. Rev. Lett. 59, 2947 (1987).

[22] S. Osnaghi, P. Bertet, A. Auffeves, P. Maioli, M. Brune, J. M. Raimond, and S. Haroche, Phys. Rev. Lett 87, 037902 (2001).

[23] I. M. Beterov and I. I. Ryabtsev, JETP Lett. 69, 448 (1999).

[24] S. Lloyd, Phys. Rev. Lett. 75, 346 (1995).

[25] A. Rauschenbeutel, G. Nogues, S. Osnaghi, P. Bertet, M. Brune, J. M. Raimond, and S. Haroche, Phys. Rev. Lett. 83, 5166 (1999).

[26] M. L. Zimmerman, M. G. Littman, M. M. Kash, and D. Kleppner, Phys. Rev. A 20, 2251 (1979).

[27] A. R. Edmonds, J. Picart, N. Tran-Minh, and R. Pullen, J. Phys. B 12, 2781, (1979).

[28] J. M. Raimond, M. Brune, and S. Haroche, Rev. Mod. Phys. 73, 565 (2001).

[29] I. I. Ryabtsev and D. B. Tretyakov, Phys. Rev. A 64, 033413 (2001).

[30] I. I. Ryabtsev and I. M. Beterov, Phys. Rev. A 61, 063414 (2000).

[31] I. I. Ryabtsev, D. B. Tretyakov, and I. I. Beterov, J. Phys. B 36, 297 (2003).

[32] C. E. Theodosiou, Phys. Rev. A 30, 2881 (1984). 